\title{
The Artificial Sky Brightness in Europe Derived from DMSP Satellite Data.
}

\author{
P. Cinzano ${ }^{1}$ and F. Falchi
}

Dipartimento di Astronomia, Università di Padova, vicolo dell'Osservatorio 5, I-35122 Padova, Italy

\author{
C. D. Elvidge and K. E. Baugh
}

Solar-Terrestrial Physics Division, NOAA National Geophysical Data Center, 3100 Marine Street, Boulder CO 80303

\begin{abstract}
We present maps of the artificial sky brightness in Europe in $V$ band with a resolution of $\sim 1 \mathrm{~km}$. The aim is to understand the state of night sky pollution in Europe, to quantify the present situation and to allow future monitoring of trends. For each terrestrial site the artificial sky brightness in a given direction on the sky is obtained by integrating the contributions from each surface area in the surroundings, using detailed models of the propagation in the atmosphere of the upward light flux emitted by the area. The top-of-atmosphere light flux is measured by the Operational Linescan System of the Defence Meteorological Satellite Program (DMSP) satellites. The modelling technique, which was introduced and developed by Garstang, takes into account the extinction along light paths, double scattering of light from atmospheric molecules and aerosols, and Earth curvature. Use of this technique allows us to assess the aerosol content of the atmosphere.
\end{abstract}

\section{Introduction}

An effective battle against light pollution requires knowledge of the situation of the night sky in large territories, recognition of the worst affected areas, determination of the growth trends and the identification of the more polluting cities. Therefore a method of mapping the artificial sky brightness over large territories is required. This is also useful in order to recognize less polluted areas and potential astronomical sites.

The DMSP satellites provide direct information on the upward light emission from almost all countries around the World (Sullivan 1989; Elvidge et al. 1997a, 1997b, 1997c, 1999; Isobe \& Hamamura 1998). We present the outlines of a method to map the artificial sky brightness in large territories using the upward flux measured directly in DMSP satellite night-time images. The method avoids errors which arise when population data are used to estimate upward

\footnotetext{
${ }^{1}$ email: cinzano@pd.astro.it
} 
flux. The effects of light pollution are computed using a detailed model of light pollution propagation in the atmosphere. Details are extensively discussed by Cinzano et al. (2000), where maps for $B$ band are also presented.

\section{Satellite Data}

U.S. Air Force Defense Meteorological Satellite Program (DMSP) satellites are in low altitude $(830 \mathrm{~km})$ sun/synchronous polar orbits with an orbital period of 101 minutes. With 14 orbits per day they generate a global nightime and daytime coverage of the Earth every 24 hours. The Operational Linescan System (OLS) is an oscillating scan radiometer with low-light visible and thermal infrared imaging capabilities. At night the instrument for visible imagery is a Photo Multiplier Tube (PMT) sensitive to radiation from $485 \mathrm{~nm}$ to $765 \mathrm{~nm}$ (FWHM), with the highest sensitivity at $550-650 \mathrm{~nm}$ where the most widely used lamps for external night-time lighting have their strongest emission. Most of the data received by the National Oceanic and Atmospheric Administration (NOAA) National Geophysics Data Center (NGDC), which has archived DMSP data since 1992, are smoothed by on-board averaging of $5 \times 5$ adjacent detector pixels and have a nominal space resolution of $2.8 \mathrm{~km}$.

In three observational runs made during the darkest portions of the lunar cycles during March 1996 and January and February of 1997, NGDC acquired OLS data at reduced gain settings in order to avoid saturation produced in normal-gain operations in a large number of pixels inside cities due to the high OLS-PMT sensivity. Three different gain settings were used on alternating nights to overcome the dynamic range limitations of the OLS. With these data a cloud-free radiance calibrated composite image of the Earth has been obtained (Elvidge et al. 1999). The temporal compositing makes it possible to remove noise and lights from ephemeral events such as fire and lightning. The main steps in generating the night-time lights product are: 1) establishment of a reference grid with finer spatial resolution than the input imagery; 2) identification of the cloud-free section of each orbit based on OLS thermal band data; 3 ) identification of lights and removal of noise and solar glare; 4) projection of the lights from cloud-free areas from each orbit into the reference grid, with calibration to radiance units; 5) tallying of the total number of light detections in each grid cell and calculation of the average radiance value; 6) filtering images based on frequency of detection to remove ephemeral events. The final image was transformed in a latitude/longitude projection with $30^{\prime \prime} \times 30^{\prime \prime}$ pixel size. The map of Europe was obtained with a portion of $4800 \times 4800$ pixels of this final image, starting at longitude $10^{\circ} 30^{\prime}$ west and latitude $72^{\circ}$ north.

\section{Mapping Technique}

Scattering from atmospheric particles and molecules spreads the light emitted upward by the sources. If $e(x, y)$ is the upward emission per unit area in $(x, y)$, the total artificial sky brightness in a given direction of the sky in a site in $\left(x^{\prime}, y^{\prime}\right)$ is:

$$
b\left(x^{\prime}, y^{\prime}\right)=\iint e(x, y) f\left((x, y),\left(x^{\prime}, y^{\prime}\right)\right) d x d y
$$


where $f\left((x, y),\left(x^{\prime}, y^{\prime}\right)\right)$ gives the artificial sky brightness per unit of upward light emission produced by unit area in $(x, y)$ in the site in $\left(x^{\prime}, y^{\prime}\right)$. The light pollution propagation function $f$ depends in general on the geometrical disposition (altitude of the site and the area, and their mutual distance), on the atmospheric distribution of molecules and aerosols and their optical characteristics in the choosen photometric band, on the shape of the emission function of the source and on the direction of the sky observed. In some works this function has been approximated with a variety of semi-empirical propagation laws: the Treanor Law (Treanor 1973; Falchi 1998; Falchi \& Cinzano 2000), the Walker Law (Walker 1973), the Berry Law (Berry 1976) and the Garstang Law (Garstang 1991b). However, all of them ignore the effects of Earth curvature, that cannot be neglected in accurate mapping of large and non-isolated territories.

We obtained the propagation function $f\left((x, y),\left(x^{\prime}, y^{\prime}\right)\right)$ for each pair of points $(x, y)$ and $\left(x^{\prime}, y^{\prime}\right)$ using detailed models for the light propagation in the atmosphere based on the modelling technique introduced and developed by Garstang $(1986,1987,1988,1989 a, 1989 b, 1991 a, 1991 b, 1991 c, 2000)$ and also applied by Cinzano (2000a,b,c). The models assume Rayleigh scattering by molecules and Mie scattering by aerosols and take into account extinction along the light path and Earth curvature. They allow association of the predictions with well-defined parameters related to the aerosol content, so the atmospheric conditions to which predictions refer can be well known. The resolution of the maps depends on the results of integrating over a large zone: it is better than the resolution of the original images and is generally of the order of the distance between two pixel centers (less than $1 \mathrm{~km}$ ). However where sky brightness is dominated by contributions of the nearest land areas, effects of the resolution of the original image could become relevant.

We assumed the atmosphere to be in hydrostatic equilibrium under gravity, with an exponential decrease of number density with height for the atmospheric haze aerosols. Measurements show that for the first $10 \mathrm{~km}$ this is a reasonable approximation. We are interested in average or typical atmospheric conditions, not particular conditions of a given night, so a detailed modelling of the local aerosol distribution at a given night is beyond the scope of this work. We neglected the presence of sporadic denser aerosol layers at various heights or at ground level, the effects of the Ozone layer and the presence of volcanic dust. We take into account changes in aerosol content following Garstang (1986), introducing a parameter $K$ which measures the relative importance of aerosol and molecules for scattering light. The adopted modelling technique allows an assessment of the atmospheric conditions for which a map is computed, giving observable quantities like the vertical extinction at sea level in magnitudes. More detailed atmospheric models could be used whenever available.

The angular scattering function for atmospheric haze aerosols can be measured easily with a number of well known remote-sensing techniques. Being interested in a typical average function, we adopted the same function used by Garstang (1991a) and we neglected geographical gradients. The normalized emission function of each area gives the relative upward flux per unit solid angle in each direction. It is the sum of the direct emission from fixtures and the reflected emission from lighted surfaces, normalized to its integral and is not known. In this paper we assumed that all land areas have the same average nor- 
malized emission function. This is equivalent to assuming that lighting habits are similar on average in each land area and that differences from the average are randomly distributed in the territory. We choose to assume this function and check its consistency with satellite measurements, rather than directly determine it from satellite measurements because at very low elevation angles the spread is much too large to constrain adequately the function shape. We adopted for the average normalized emission function the normalized city emission function from Garstang (1986).

\section{Results}

Figures 1-6 show the maps of the artificial sky brightness in Europe at sea level in $V$ band. The maps have been computed for a clean atmosphere with an aerosol clarity $K=1$, corresponding to a vertical extinction of $\Delta m=0.33$ mag in $V$ band, horizontal visibility $\Delta x=26 \mathrm{~km}$ and optical depth $\tau=0.36$. Gray levels from black to white correspond to ratios between the artificial sky brightness and the natural sky brightness of: $<0.11,0.11-0.33,0.33-1.00,1-3,3-9$ and $>9$. We limited our computations to zenith sky brightness even if our method allows determination of brightness in other directions. This would be useful to predict visibility in large territories of particular astronomical phenomena. A complete mapping of the artificial brightness of the sky of a site, like Cinzano (2000a), but using satellite data instead of population data, is possible (Cinzano \& Elvidge 2000 , in prep.).

We are more interested in understanding and comparing light pollution distributions than in predicting the effective sky brightness for observational purposes, so we computed everywhere the artificial sky brightness at sea level, in order to avoid introducing altitude effects in our maps. We will take account of altitudes in a forthcoming paper devoted to mapping the limiting magnitude and naked-eye star visibility, which requires the computation of star-light extinction and natural sky brightness for the altitude of each land area. We neglected the presence of mountains which might shield the light emitted from the sources from a fraction of the atmospheric particles along the line-of-sight of the observer. Given the vertical extent of the atmosphere with respect to the height of the mountains, the shielding is non-negligible only when the source is very near the mountain and both are quite far from the site (Garstang 1989, see also Cinzano $2000 \mathrm{a}, \mathrm{b})$. Earth curvature emphasizes this behaviour.

We calibrated the maps on the basis of both (i) accurate measurements of sky brightness together with extinction from the earth-surface and (ii) preflight radiance calibration of OLS-PMT. Map calibration based on pre-flight irradiance calibration of OLS PMT requires knowledge, for each land area, of (a) the average vertical extinction $\Delta m$ during satellite observations and (b) the relation between the radiance in the choosen photometrical band and the radiance measured in the PMT spectral sensitivity range, which depends on the emission spectra. The result of this calibration is well inside the errors of the Earth-based calibration in spite of the large uncertainties both in the extinction and in the average emission spectra. As soon as a large number of sky brightness measurements are available, better calibration will be possible.

We are preparing a World Atlas (Cinzano et al., in prep.). 


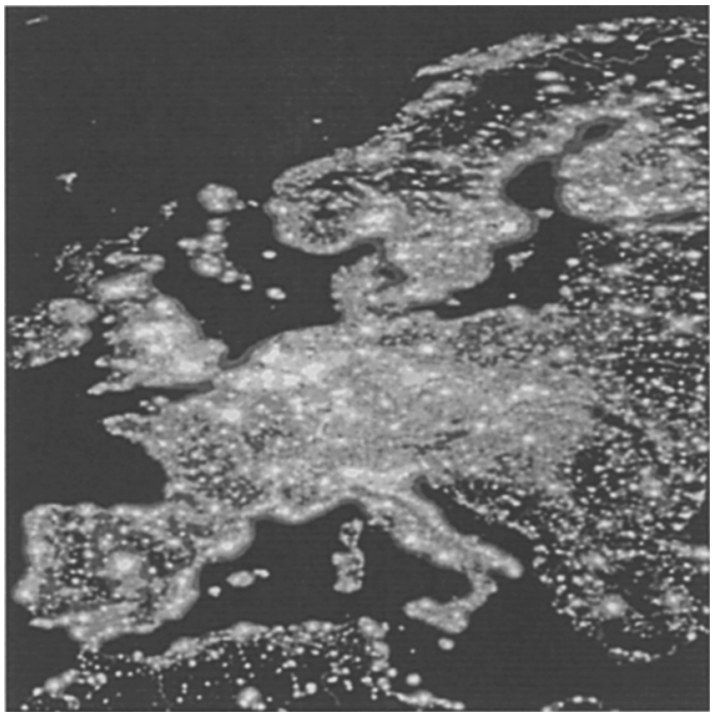

Figure 1. Artificial sky brightness at sea level in Europe in $V$ band for aerosol content parameter $K=1$.

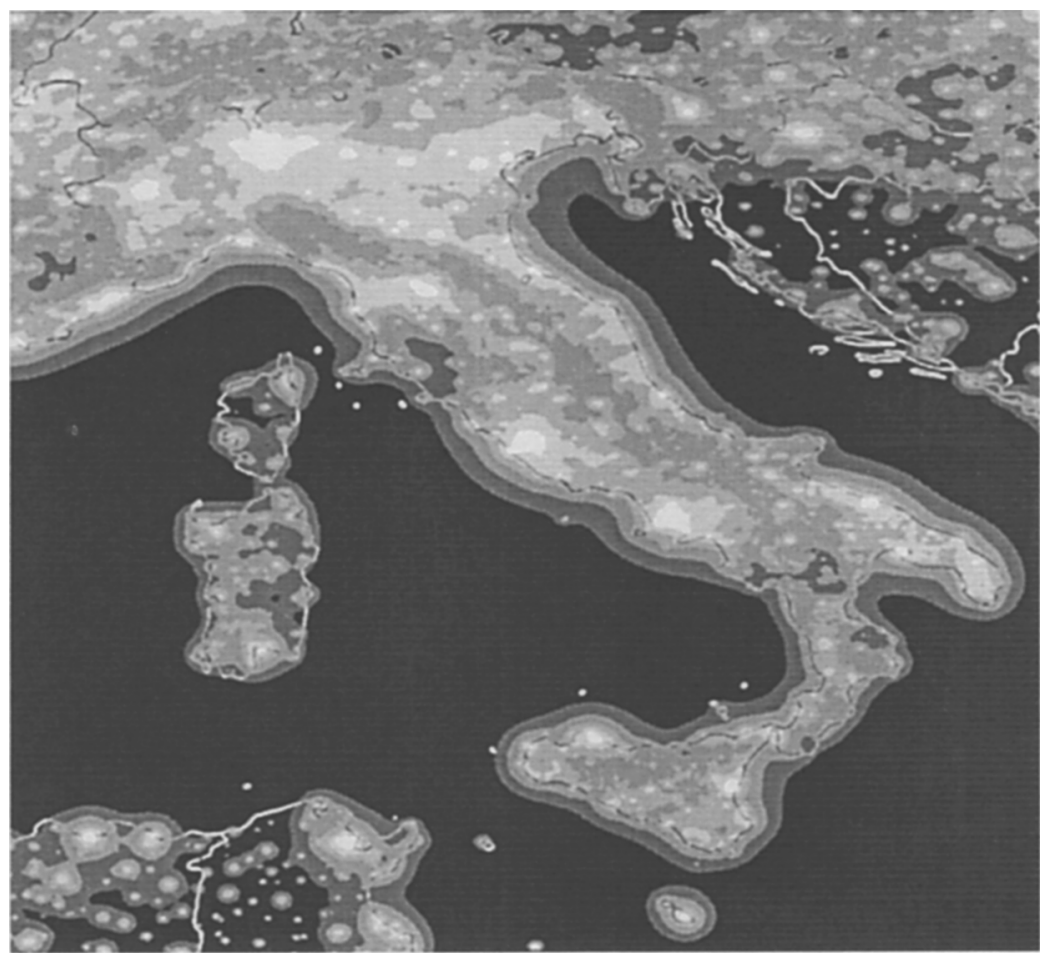

Figure 2. Artificial sky brightness at sea level in Italy in $V$ band for aerosol content parameter $K=1$. 


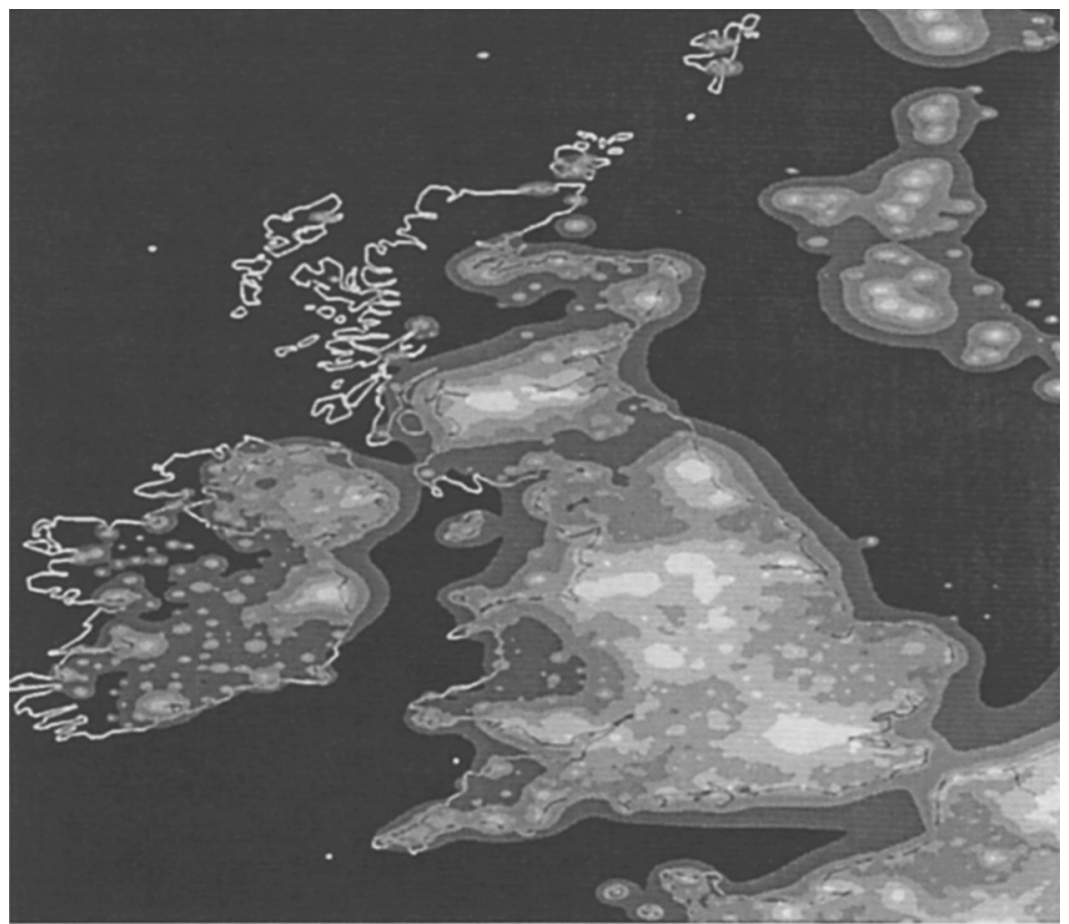

Figure 3. Artificial sky brightness at sea level in Great Britain and Ireland in $V$ band for aerosol content parameter $K=1$.

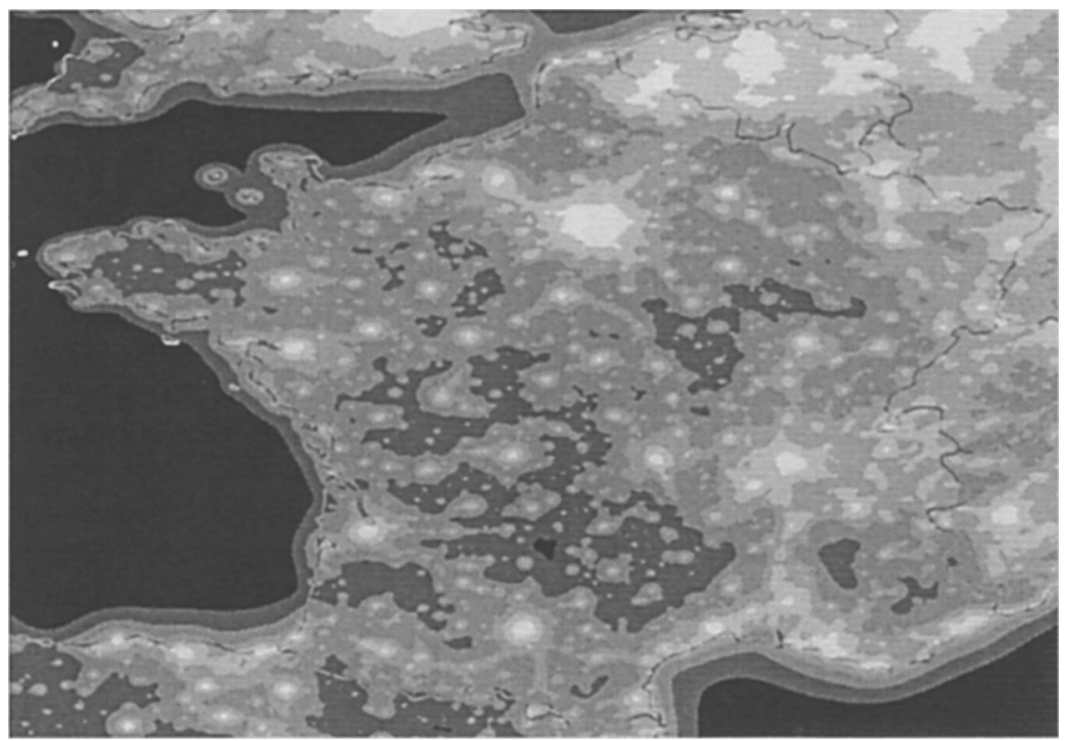

Figure 4. Artificial sky brightness at sea level in France and Belgium in $V$ band for aerosol content parameter $K=1$. 


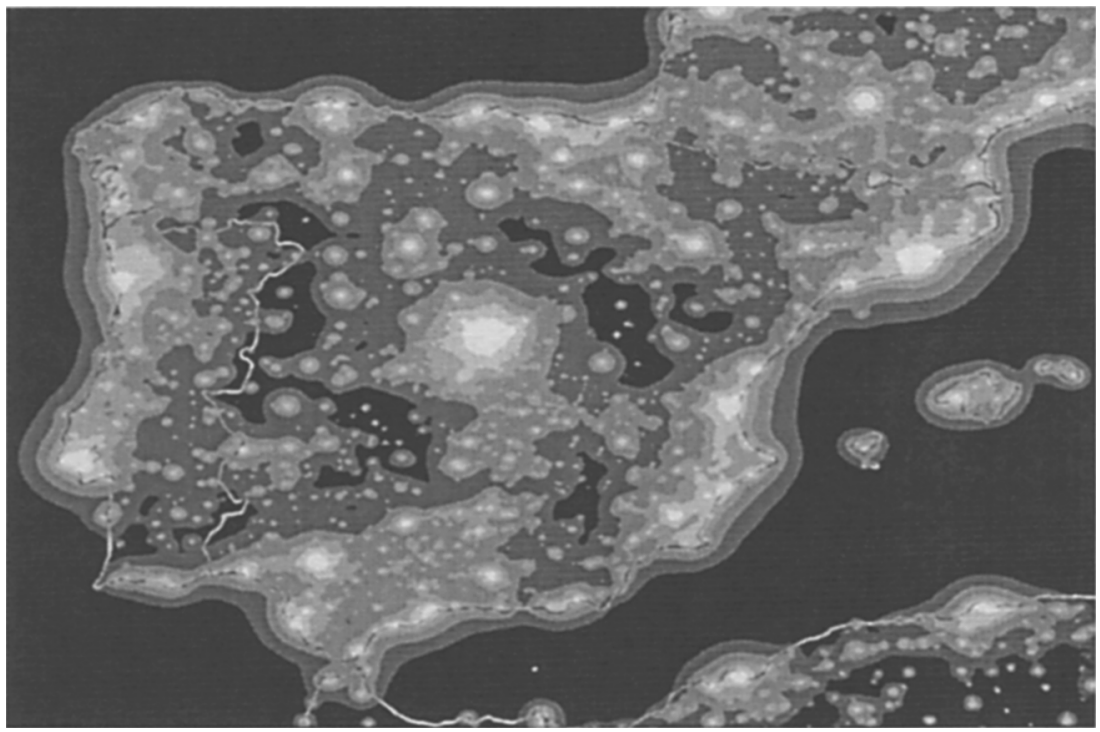

Figure 5. Artificial sky brightness at sea level in Spain and Portugal in $V$ band for aerosol content parameter $K=1$.

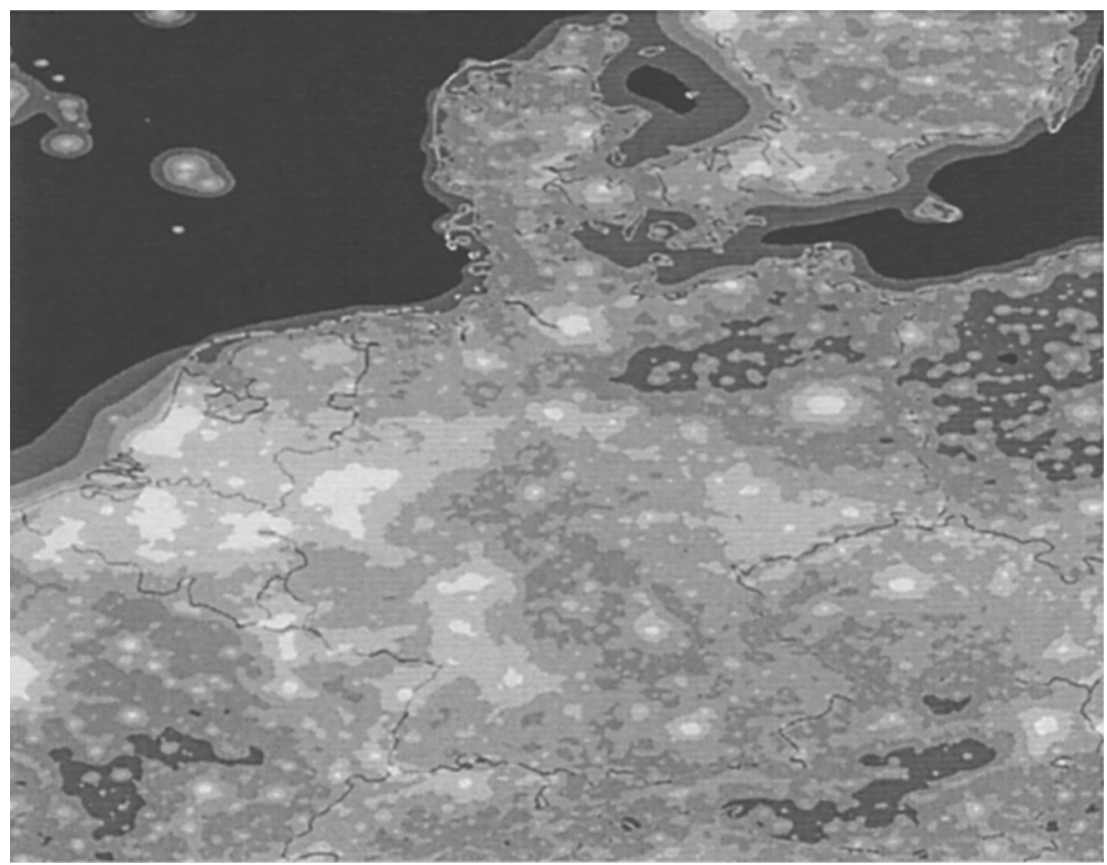

Figure 6. Artificial sky brightness at sea level in Central Europe in $V$ band for aerosol content parameter $K=1$. 
Acknowledgments. We are indebted to Roy Garstang of JILA-University of Colorado for his friendly kindness in reading and refereeing this paper, for his helpful suggestions and for interesting discussions.

\section{References}

Berry, R. 1976, J. Royal Astron. Soc. Canada, 70, 97-115

Cinzano, P. 2000a, in Measuring and Modelling Light Pollution, ed. P. Cinzano, Mem. Soc. Astron. Ital., 71, 1, 113-130

Cinzano, P. 2000b, in Measuring and Modelling Light Pollution, ed. P. Cinzano, Mem. Soc. Astron. Ital., 71, 1, 93-112

Cinzano, P. 2000c, in Measuring and Modelling Light Pollution, ed. P. Cinzano, Mem. Soc. Astron. Ital., 71, 1, 239-250

Cinzano, P., Falchi, F., Elvidge, C.D., Baugh, K.E. 2000, MNRAS, 318, 641-657

Elvidge, C.D., Baugh, K.E., Kihn, E.A., Kroehl, H.W., Davis, E.R. 1997a, Photogrammetric Engineering and Remote Sensing, 63, 727-734

Elvidge, C.D., Baugh, K.E., Kihn, E.A., Kroehl, H.W., Davis, E.R., Davis, C. 1997b, Int. J. of Remote Sensing, 18, 1373-1379

Elvidge, C.D., Baugh, K.E., Hobson, V.H., Kihn, E.A., Kroehl, H.W., Davis, E.R., Cocero, D. 1997c, Global Change Biology, 3, 387-395

Elvidge, C.D., Baugh, K.E., Dietz, J.B., Bland, T., Sutton, P.C., Kroehl, H.W. 1999, Remote Sensing of Environment, 68, 77-88

Falchi, F. 1998, Thesis, University of Milan

Falchi, F., Cinzano, P. 2000, in Measuring and Modelling Light Pollution, ed. P. Cinzano, Mem. Soc. Astron. Ita., 71, 1, 139-152

Garstang, R.H. 1986, Publ. Astron. Soc. Pacific, 98, 364-375

Garstang, R.H. 1987, in Identification, optimization and protection of optical observatory sites, eds. R.L. Millis, O.G. Franz, H.D. Ables \& C.C. Dahn (Flagstaff: Lowell Observatory), 199-202

Garstang, R.H. 1988, The Observatory, 108, 159-161

Garstang, R.H. 1989a, Publ. Astron. Soc. Pacific, 101, 306-329

Garstang, R.H. 1989b, Ann. Rev. Astron. Astrophys., 27, 19-40

Garstang, R.H. 1991a, Publ. Astron. Soc. Pacific, 103, 1109-1116

Garstang, R.H. 1991b, in Light Pollution, Radio Interference and Space Debris, IAU Coll. 112, ed. D.L. Crawford, Astron. Soc. of Pacific Conference Series 17, 56-69

Garstang, R.H. 1991c, The Observatory, 111, 239-243

Garstang, R.H. 2000, in Measuring and Modelling Light Pollution, ed. P. Cinzano, Mem. Soc. Astron. Ital., 71, 1, 71-82

Isobe S. \& Hamamura, S. 1998, in Preserving the Astronomical Windows, IAU JD5, ed. S. Isobe, Astron. Soc. of Pacific Conference Series 139, 191-199

Sullivan, W.T., 1989, Int. J. of Remote Sensing, 10, 1-5

Treanor, P.J.S.J. 1973, The Observatory, 93, 117-120

Walker, M.F. 1973, Publ. Astron. Soc. Pacific, 85, 508-519 